\title{
Enfin un modèle animal apparemment pertinent de maladie d'Alzheimer!
}

La maladie d'Alzheimer, qui fait l'objet de tant de recherches, conserve le mystère de son origine. On sait que s'accumule un peptide $\beta$ amyloïde dit $A \beta$, qui compte 42 acides aminés. Son précurseur, la protéine APP, existe en plusieurs isoformes; l'APP est codée par un gène de grande taille (environ $400 \mathrm{kpb}$ ) ; le peptide $\mathrm{A} \beta$ est formé par un processus de dégradation dans les lysosomes et les endosomes. Dans les formes familiales de maladie d'Alzheimer, on a décrit un certain nombre de mutations de l'APP $\left(m / s n^{\circ} 8\right.$, vol. 8 , p. 866), dont le gène est sur le chromosome 21 , et l'on sait que les signes de maladie d'Alzheimer apparaissent souvent précocement dans les trisomies 21 [1]. Cependant, de telles mutations sont très minoritaires $\left(\mathrm{m} / \mathrm{s} n^{\circ} 9\right.$, vol. 8 , p. 1007). La production in vivo du peptide $A \beta$ est normale et on en trouve dans le plasma et le liquide céphalo-rachidien des sujets normaux comme dans la maladie d'Alzheimer. In vitro, des cellules en culture en fabriquent [2]; de plus, la transfection avec des formes mutées d'APP montre une accumulation accrue du peptide ([3] et $m / s n^{\circ} 1$, vol. 9, p. 108). Depuis de nombreuses années, plusieurs équipes ont tenté de créer des modèles murins de maladie d'Alzheimer par transgénèse, et médecine/sciences a conté leurs déboires. Les méthodes utilisées consistaient toutes en l'introduction d'un transgène entraînant une hyperproduction de différentes régions de la protéine $\beta$ amyloïde dans des cerveaux murins $\left(m / s n^{\circ} 8\right.$, vol. 7, p. 859). Certains de ces gènes codant pour la protéine APP $\beta$ amyloid precursor protein) reproduisaient la séquence humaine normale, d'autres $m / s n^{\circ} 4$, vol. 11, avril 95 comportaient les mutations de ce gène associées chez l'homme à des formes génétiques de maladie d'Alzheimer. Cependant, par souci de simplicité, ces essais antérieurs utilisaient souvent des molécules d'ADNc [4]. Or, pour des raisons qui semblent encore peu claires, on sait que la présence d'introns améliore le plus souvent considérablement (souvent d'un facteur 10 à 100) le niveau d'expression de transgènes. Games et al. (34 auteurs de différents laboratoires américains des côtes Est et Ouest) [5] ont utilisé une construction particulière constituée de l'ADNc des exons 1 à 6 , la région génique allant de l'intron 6 à l'intron 8, et enfin l'ADNc de l'exon 9 à l'exon 18. Cette construction était placée sous le contrôle des séquences régulatrices $\mathrm{du}$ PDGF $\beta$ (platelet derived growth fac$\operatorname{tor} \beta$ ). Ce minigène porte la mutation Val 717 Phe dans le seizième exon. Les séquences régulatrices du gène du PDGF $\beta$ sont connues comme étant particulièrement actives dans les neurones du cortex, de l'hippocampe, de l'hypothalamus, du corps calleux et du cervelet d'animaux transgéniques, certaines de ces régions étant électivement atteintes dans la maladie d'Alzheimer (par exemple, le cortex et l'hippocampe). La séquence génique entre les introns 6 et 8 permet l'épissage alternatif physiologique engendrant les trois formes normales d'APP. En effet, l'expression chez certains des animaux transgéniques obtenus, contenant un grand nombre de copies de transgènes intégrés, est très élevée, avec présence des trois formes de transcrits attendues. A partir de 6-9 mois, les animaux commencent à accumuler des dépôts de peptides amyloïdes dans l'hippocampe, le corps calleux et le cortex cérébral. Ces lésions se complètent progressivement, aboutissant à la présence des signes anatomo-pathologiques typiques de la maladie d'Alzheimer chez l'homme: dépôts extracellulaires du peptide $\beta$ amyloïde, plaques neuritiques, perte de densité synaptique, astrocytose et microgliose. Naturellement, il est difficile, chez la souris, de repérer les signes cliniques les plus caractéristiques de la maladie d'Alzheimer: troubles de la mémoire et du comportement, démence, etc. Néanmoins, des études neuro-comportementales fines devraient permettre d'identifier l'éventuelle présence chez ces animaux de troubles fonctionnels associés aux anomalies histologiques. De plus, l'effet de différents médicaments sur la progression des lésions pourra probablement être étudié. Par exemple, Kisilevsky et al. (Kingston, Canada) viennent de montrer que de petites molécules anioniques à radicaux sulfate ou sulfonate pouvaient inhiber in vivo le dépôt intrasplénique de substance amyloïde associé à l'inflammation (AA) [6]. In vitro, ces agents inhibent également l'agrégation du peptide $\beta$ amyloïde. Il sera donc particulièrement intéressant de tester de tels composés sur la formation des dépôts amyloïdes chez les souris de Games et al. [5].

Ce modèle pourrait également donner de précieux renseignements sur la physiopathologie des signes anatomopathologiques et neurologiques. On sait que plusieurs mécanismes ont été proposés. L'un d'entre eux, peut-être celui qui a aujourd'hui le plus de déf enseurs, repose sur la neurotoxicité directe du peptide $\beta$ amy. 
loïde $\left(m / s n^{\circ} 2\right.$, vol. 8 , p. 180). Celle-ci vient récemment d'être confirmée par les travaux de LaFerla et al. (Rockville, MD, USA) qui ont créé des souris transgéniques exprimant une construction dans laquelle la séquence du peptide $\beta$ amyloïde dépourvu de peptide signal a été placée sous le contrôle du promoteur du gène codant pour la chaîne légère des neurofilaments (NF-L), spécifiquement et fortement exprimé dans les neurones [7]. La synthèse active de peptide $\beta$ amyloïde et son accumulation intracellulaire provoquent une importante mort neuronale par apoptose, avec gliose réactionnelle. Les souris souffrent de crises épileptoïdes suivies de léthargie et $50 \%$ d'entre elles sont mortes à un an alors que, dans la souche utilisée, la survie normale est de deux ans.

Le second mécanisme souvent évoqué passe par la formation des faisceaux neurofibrillaires au niveau desquels s'accumule la protéine tau hyperphosphorylée [8]. La protéine tau stabilise les microtubules, fonction qui pourrait être perturbée par son hyperphosphorylation, aboutissant à de graves perturbations neuronales, notamment des transports axonaux. L'hyperphosphorylation elle-même a été récemment attribuée à une diminution de l'activité des phosphatases cellulaires, dont rien ne dit qu'elle n'est pas en fait secondaire à des lésions neuronales d'autre nature. Quoi qu'il en soit, selon ce mécanisme, la protéine tau hyperphosphorylée serait un acteur important de la constitution des lésions, agissant peut-être de concert avec un troisième agent étiologique souvent invoqué, l'aluminium. L'aluminium, en effet, semble se complexer avec la protéine tau hyperphosphorylée pour former des agrégats résistant à la protéolyse [8] C'est dire que les conclusions seront toutes différentes selon qu'apparaîtront spontanément des faisceaux neurofibrillaires chez les souris de Games et al. [5], ou bien que ce signe manquera. Une étude de l'éventuel effet de l'aluminium chez ces animaux pourrait aussi éclairer une pathogénie encore incertaine, surtout si ce métal permettait de faire apparaître les faisceaux neurofibril- laires. Des croisements avec des souris hyperexprimant les apolipoprotéines facteurs de susceptibilité à la maladie d'Alzheimer (Apo E4, $\mathrm{m} / \mathrm{s}$ $n^{\circ}$ 10, vol. 9, p. 1142) ou facteurs de protection (ApoE2, $m / s n^{\circ} 8 / 9$, vol.10, p. 920) pourraient aussi permettre de confirmer les hypothèses actuelles sur l'interaction entre ces apolipoprotéines et la protéine $\beta$ amyloide dans l'apparition des lésions et des symptômes.

J.C.D.

A.K.

1. Sinet P, Nicolle A, Ceballos I, Delabar J. Trisomie 21 et maladie d'Alzheimer. médecine/sciences 1987; 3 : 256-62.

2. Haass C, Schlossmacher MG, Hung AY, VigoPelfrey C, Mellon A, Ostaszewski BL, Lieberburg I, Koo EH, Schenk D, Teplow DB, Selkoe DJ. Amyloid $\beta$ peptide is produced by cultured cells during normal metabolism. Nature 1992; 359 : 322-5.

3. Cai XD, Golde TE, Youkin SG. Release of excess amyloid $\beta$ protein from a mutant amyloid $\beta$ protein precursor. Science 1993; 259: 514-6.

4. Lamb BT Making models for Alzheimer's disease. Nature Genet 1995; 9: 4-6.

5. Games D, Adams D, Allessandrini R, et al . Alzheimer-type neuropathology in transgenic mice overexpressing V717F, $\beta$ amyloid precursor protein. Nature $1995 ; 373$ : 523-7

6. Kisilevsky R, Lemieux LJ, Fraser PE, Kong X, Hultin PG, Szarek WA. Arresting amyloidosis in vivo using small-molecule anionic sulphonates or sulphates: implications for Alzheimer's disease. Nature Med 1995; 1 : 143-8.

7. LaFerla FM, Tinkle BT, Bieberich J, Haudenschild CC, Jay G. The Alzheimer's A $\beta$ peptide induces neurodegeneration and apoptotic cell death in transgenic mice. Nature Genet 1995; 9 ; 21-9

8. Roush W. Protein studies try to puzzle out Alzheimer's tangles. Science 1995; 267 : 793-4 